\title{
Multiple blood pressure loci with opposing blood pressure effects on rat chromosome 1 in a homologous region linked to hypertension on human chromosome 15
}

\author{
Blair Mell, Shakila Abdul-Majeed, Sivarajan Kumarasamy, Harshal Waghulde, Resmi Pillai, Ying Nie \\ and Bina Joe
}

Genetic dissection of blood pressure (BP) quantitative trait loci (QTLs) in rats has facilitated the fine-mapping of regions linked to the inheritance of hypertension. The goal of the current study was to further fine-map one such genomic region on rat chromosome 1 (BPQTL1b1), the homologous region of which on human chromosome 15 harbors BP QTLs, as reported by four independent studies. Of the six substrains constructed and studied, the systolic BP of two of the congenic strains were significantly lower by 36 and $27 \mathrm{~mm} \mathrm{Hg}$ than that of the salt-sensitive (S) rat $(P<0.0001, P=0.0003$, respectively). The congenic segments of these two strains overlapped between 135.12 and $138.78 \mathrm{Mb}$ and contained eight genes and two predicted miRNAs. None of the annotations had variants within expressed sequences. These data taken together with the previous localization resolved QTL1b1 with a $70 \%$ improvement from the original $7.39 \mathrm{Mb}$ to the current $2.247 \mathrm{Mb}$ interval. Furthermore, the systolic BP of one of the congenic substrains was significantly higher by $20 \mathrm{~mm} \mathrm{Hg}(P<0.0001)$ than the BP of the S rat. The limits of this newly identified QTL with a BP increasing effect (QTL1b1a) were between 134.12 and $135.76 \mathrm{Mb}$, spanning $1.64 \mathrm{Mb}$, containing two protein-coding genes, Mctp2 and Rgma, and a predicted miRNA. There were four synonymous variants within Mctp2. These data provide evidence for two independent BP QTLs with opposing BP effects within the previously identified BP QTL1b1 region. Additionally, these findings illustrate the complexity underlying the genetic mechanisms of BP regulation, wherein inherited elements beyond protein-coding sequences or known regulatory regions could be operational.

Hypertension Research (2015) 38, 61-67; doi:10.1038/hr.2014.134; published online 18 September 2014

Keywords: BP QTL; Dahl S rat; HSA15; RNO1

\section{INTRODUCTION}

Four independent studies have detected linkage of human essential hypertension to a large region encompassing the q-terminus of human chromosome $15 .^{1-4}$ Using homology mapping, this region in humans can be located on rat chromosome 1 (RNO1) wherein multiple previous linkage and substitution mapping studies have reported blood pressure (BP) quantitative trait loci (QTLs). ${ }^{5-10}$ Among these, a 7.4-Mb genomic segment on RNO1 was previously identified as BP QTL1b1 using congenic strains developed by introgressing alleles from the normotensive Lewis (LEW) rat into the genome of the hypertensive salt-sensitive (S) rat. ${ }^{11}$ LEW alleles within this QTL lowered BP of the S rat. QTL1b1 was defined by the limits of the RNO1 congenic strain S.LEW(D1Mco36-D1Mco101) ${ }^{11}$ (Figure 1). The purpose of the current study was to further resolve this BP QTL.
A panel of six congenic substrains was constructed and their BP phenotypes were compared with that of the $S$ rat. Data obtained from these strains clearly indicate that there are two BP QTLs. These two BP QTLs are named QTL1b1 and QTL1b1a. LEW alleles of QTL1b1 decreased the BP of the $\mathrm{S}$ rat and were mapped within $2.247 \mathrm{Mb}$ containing 17 annotations. LEW alleles of QTL1bla increased the BP of the $\mathrm{S}$ rat and were fine-mapped within $1.64 \mathrm{Mb}$ containing five annotations. Genomic DNA sequencing data from these mapped locations were cataloged. While there were several intronic and intergenic polymorphisms, there were neither any non-synonymous variants nor any detectable alterations in the gene expression of candidate protein-coding genes between the strains. Collectively, these data point to variants outside protein-coding elements as likely candidates for the two BP QTLs with opposing BP effects.

Department of Physiology and Pharmacology, Center for Hypertension and Personalized Medicine, Program in Physiological Genomics, University of Toledo College of Medicine and Life Sciences, Toledo, OH, USA

Correspondence: Professor B Joe, Department of Physiology and Pharmacology, Center for Hypertension and Personalized Medicine, Program in Physiological Genomics, University of Toledo College of Medicine and Life Sciences, 3000 Arlington Avenue, Toledo, OH 43614-2598, USA.

E-mail: bina.joe@utoledo.edu

Received 10 June 2014; revised 7 July 2014; accepted 9 July 2014; published online 18 September 2014 


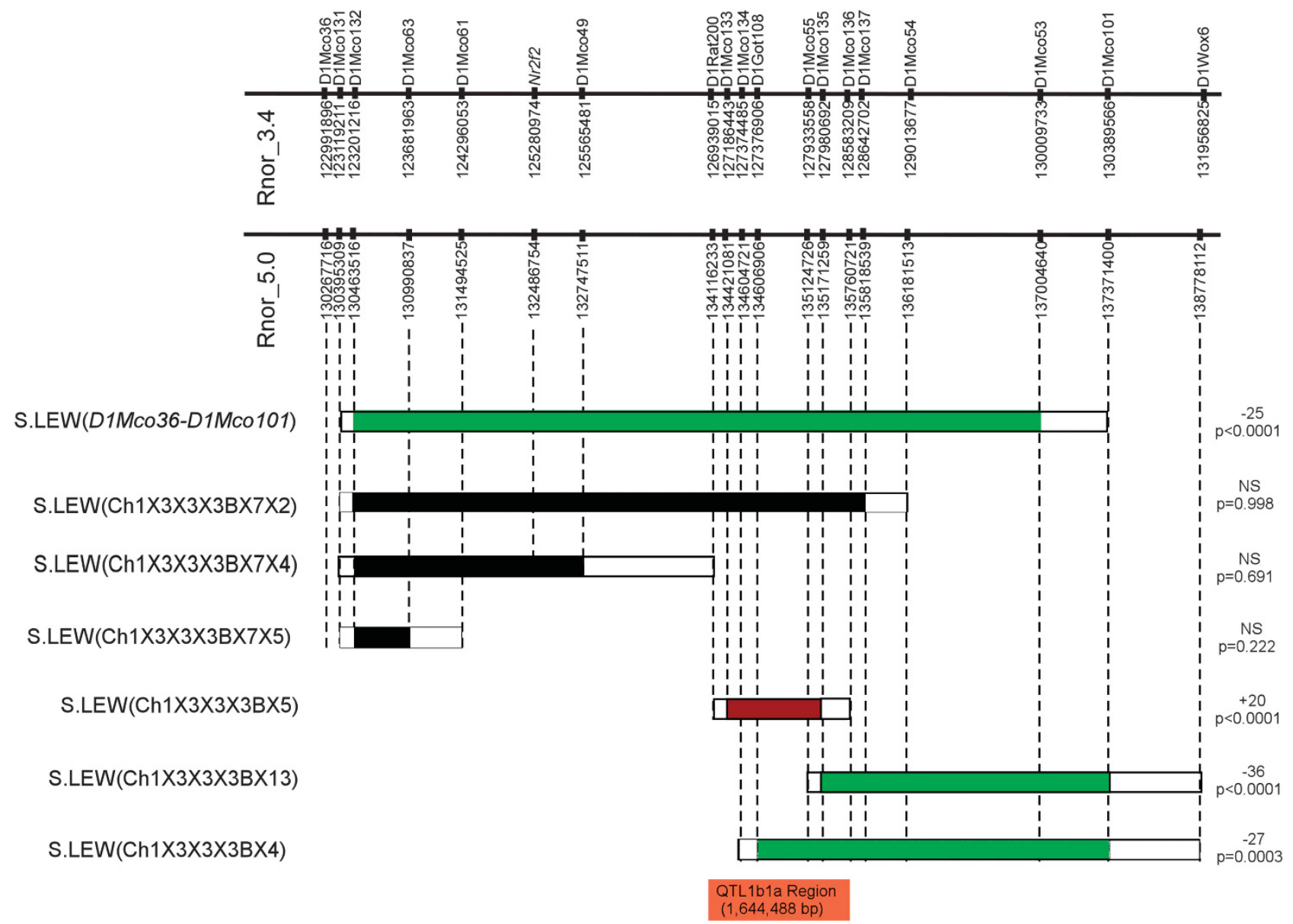

QTL1b1 Region $(2,246,674 \mathrm{bp})$

Figure 1 The physical map of RNO1 along with the microsatellite markers and their locations according to the Ensembl database (www.ensembl.org, RGSC 3.4 and 5.0) are depicted. The horizontal bars alongside of the physical map represent schematics of congenic strains. Colored boxes are introgressed LEW segments. A green bar indicates that the congenic strain had a lower BP than S. A black bar indicates that the congenic strain had no significant change in BP compared with S. A red bar indicates that the congenic strain had a higher BP than S. The regions of recombination are represented by white boxes flanking each of the congenic strains. The BP effect of each strain compared with $\mathrm{S}$ is shown to the right of the schematic of each congenic strain. BP effect and $P$-values for the bottom three strains are from telemetry experiments. The locations of the newly identified BP QTLs are indicated as orange bars at the bottom of the figure. Data from S.LEW(D1Mco36-D1Mco101) was previously published ${ }^{11}$ and is presented here for completeness.

Table 1 Systolic blood pressures of congenic strains and S obtained by the tail-cuff method

\begin{tabular}{|c|c|c|c|c|}
\hline Congenic strain & $\begin{array}{l}\text { Systolic } \\
\text { BP of S }\end{array}$ & $\begin{array}{l}\text { Systolic BP } \\
\text { of congenic }\end{array}$ & $\begin{array}{c}\text { BP effect } \\
\text { (congenic } \\
\text { minus S, } \\
m m \mathrm{Hg} \text { ) }\end{array}$ & P-value \\
\hline S.LEW(D1Mco36-D1Mco101)a & $198 \pm 2$ & $173 \pm 1$ & -25 & $<0.0001$ \\
\hline 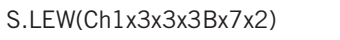 & $226 \pm 4$ & $226 \pm 5$ & 0 & 0.998 \\
\hline S.LEW(Ch $1 \times 3 \times 3 \times 3 B \times 7 \times 4)$ & $217 \pm 5$ & $226 \pm 4$ & +9 & 0.691 \\
\hline 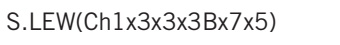 & $208 \pm 6$ & $227 \pm 7$ & +19 & 0.222 \\
\hline 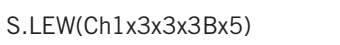 & $194 \pm 5$ & $212 \pm 5$ & +18 & 0.014 \\
\hline 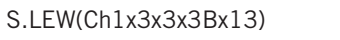 & $198 \pm 8$ & $157 \pm 6$ & -41 & $<0.0001$ \\
\hline 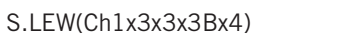 & $198 \pm 8$ & $155 \pm 16$ & -43 & 0.009 \\
\hline
\end{tabular}

Abbreviation: BP, blood pressure; LEW, Lewis; S, salt-sensitive.

aData from this strain were previously published ${ }^{11}$ and is presented here for completeness. Systolic BP of S given in each row is the value obtained with the concomitantly raised group of $S$ rats along with the test congenic strain. Same value for the $S$ column indicates that the same group of $\mathrm{S}$ rats were raised and tested along with two different congenic strains. Values are mean \pm s.e. of the mean

\section{METHODS}

\section{Animals}

All animal research protocols were approved by the University of Toledo's Institutional Animal Care and Use Committee. Experiments were conducted in accordance with the National Institutes of Health Guide for the Care and Use of Laboratory Animals. Dahl salt-sensitive (SS/Jr or S) rats were inbred in our colony. The Lewis (LEW/NCrlBR) rats, originally obtained from Charles River Laboratories (Wilmington, MA, USA), were maintained in our animal facility and are referred to as LEW. All congenic substrains were constructed as per previously published procedures. $^{9}$ S.LEW(D1Mco36-D1Mco101) was the progenitor strain for all strains in the study.

\section{Microsatellite markers}

New microsatellite markers were developed from sequences identified by searching the rat genome sequence data available (RGSC 3.4 or RGSC 5.0) through the Ensembl database (http://www.ensembl.org). Marker names and details are deposited with the Rat Genome Database (http://rgd.mcw.edu).

\section{Genotyping}

Congenic substrain DNA was extracted from a tail biopsy using the Promega Wizard SV 96 Genomic DNA Purification System (Promega, Madison, WI, 


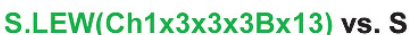
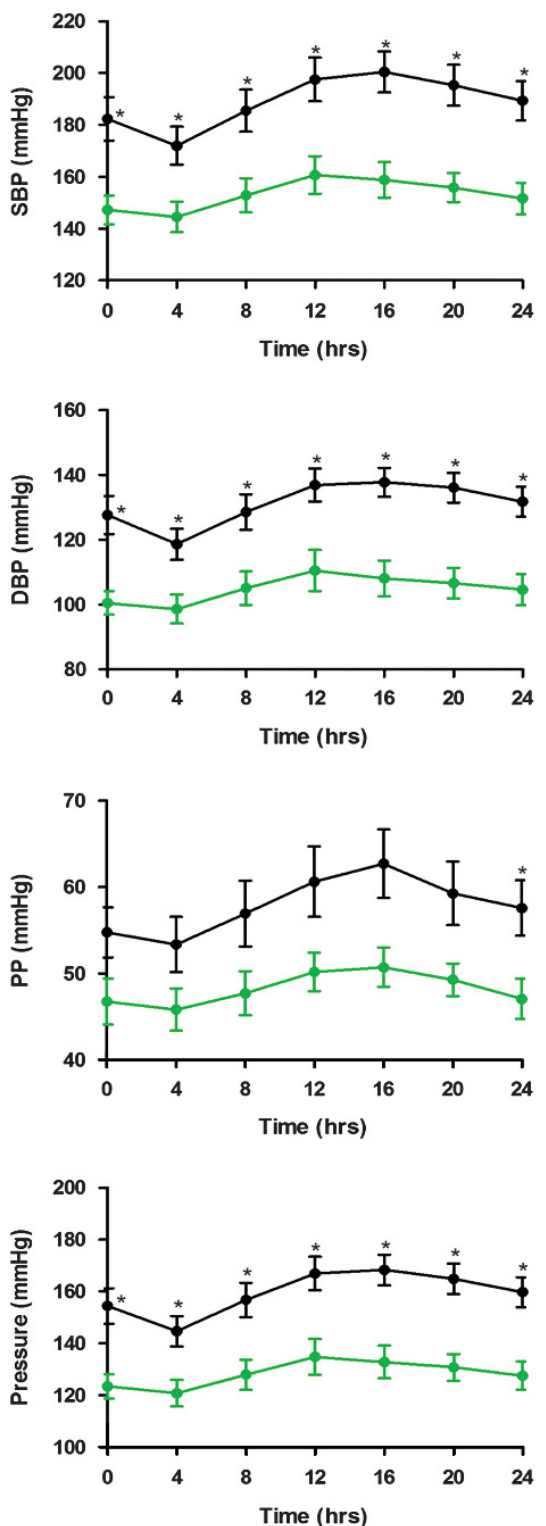

S.LEW(Ch1 $1 \times 3 \times 3 \times 3 B \times 4)$ vs. S
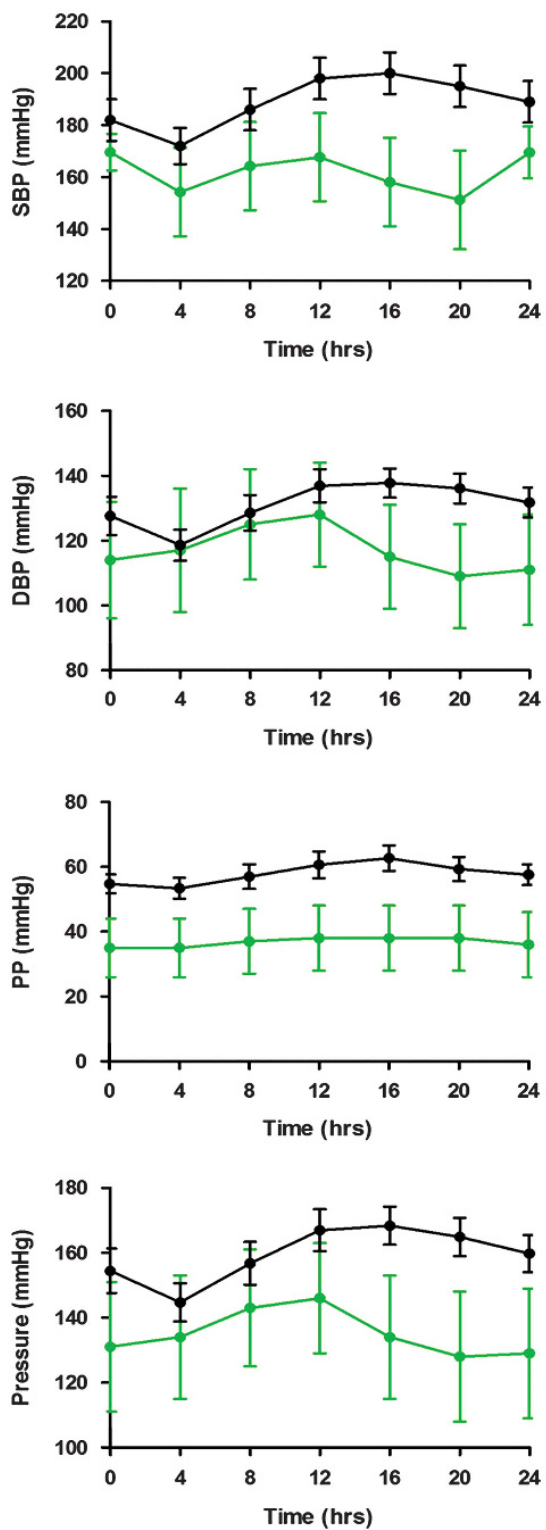

S.LEW(Ch1 $1 \times 3 \times 3 \times 3 B \times 5)$ vs. S
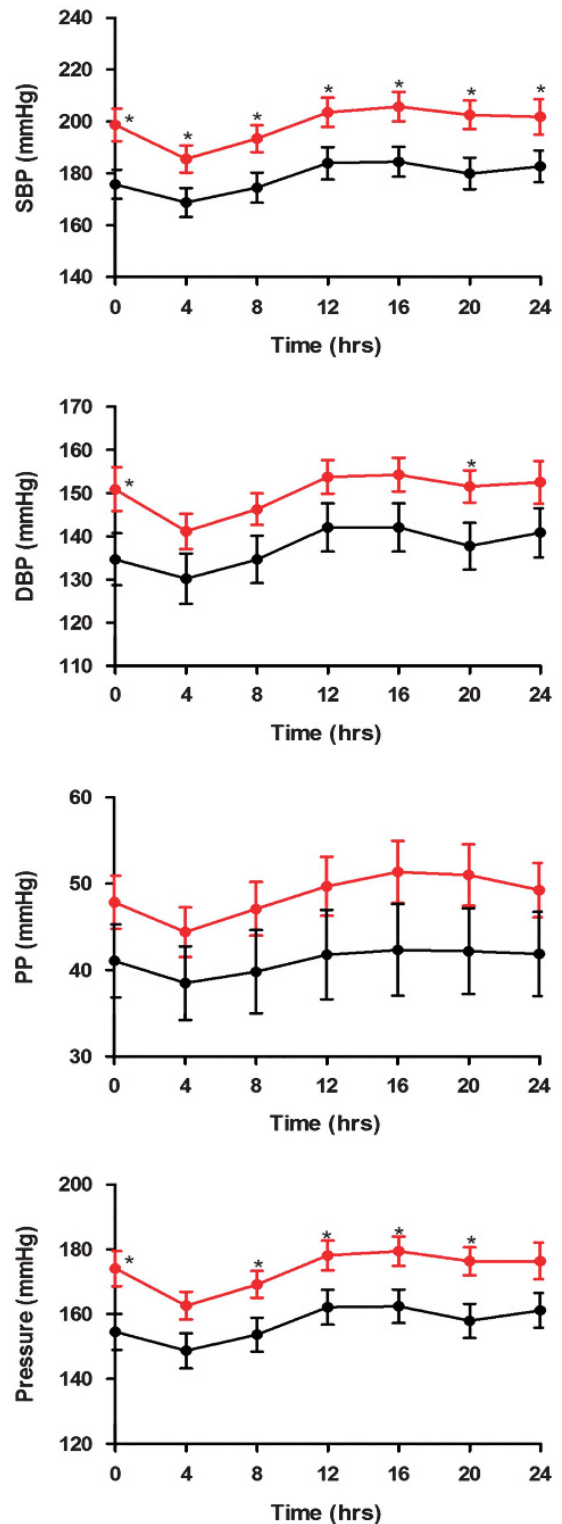

Figure 2 Blood pressure measurements by radiotelemetry. $S$ and congenic strains were implanted with radio-transmitters and their BP was continuously recorded for $24 \mathrm{~h}$ as detailed under the Methods. $\mathrm{N}=10-12$ rats/group. SBP, systolic blood pressure; DBP, diastolic blood pressure; PP, pulse pressure; Pressure, mean blood pressure.

USA). PCR-based genotyping using microsatellite markers was performed using standard techniques as described previously. ${ }^{12}$

\section{Phenotyping}

Rats were weaned at 28-30 days of age and maintained on a low-salt $(0.3 \%$ $\mathrm{NaCl}$ ) Harlan Teklad diet, 7034 (Madison, WI, USA). For each congenic strain, 20-30 male congenic substrain rats were raised concomitantly with male $S$ control rats and matched by weight as closely as possible with the $\mathrm{S}$ rats for BP studies. Each cage contained a congenic strain and an $S$ rat. In studies comparing two congenic substrains with $\mathrm{S}$, one congenic rat was housed with either an $\mathrm{S}$ rat or another congenic rat representing a different congenic strain. The rats were fed a $2 \% \mathrm{NaCl}$ Harlan Teklad diet, TD94217, for 24 days starting at 40-42 days of age. The tail-cuff method was used to measure the systolic BPs of conscious restrained rats warmed to $28^{\circ} \mathrm{C}$ using equipment from IITC (Woodland Hills, CA, USA). BP was measured once a day for 4 consecutive days during the same time period (morning). An average BP reading for each day was taken from two to three consistent readings. The final BP was calculated from the average BP value of the 4 days. At the end of the study, rats were euthanized by $\mathrm{CO}_{2}$, and final body and heart weights were recorded. If a change in $\mathrm{BP}$ was observed between the congenic rats and the $\mathrm{S}$ rats, then the $\mathrm{BP}$ of a smaller subset of 10-12 animals/group of congenic and $\mathrm{S}$ rats was subsequently tested further via radiotelemetry post-surgical implantation of C-40 transmitters (Data Sciences International, St Paul, MN, USA) as described previously. ${ }^{7}$ After 5 days of recovery from surgery, the transmitters were turned on and systolic BP, diastolic BP, mean BP, heart rate and pulse pressure were recorded for 4 consecutive days. The average BP of each 4-h time interval was used for statistical analysis. Statistical analyses were performed as previously reported. ${ }^{11}$

\section{Quantitative real-time PCR}

Heart and kidney RNA samples from 10-week-old S $(n=5$ heart, $n=5$

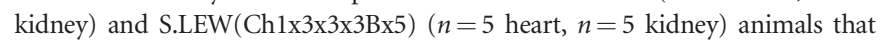


Table 2a Annotations within QTL1b1

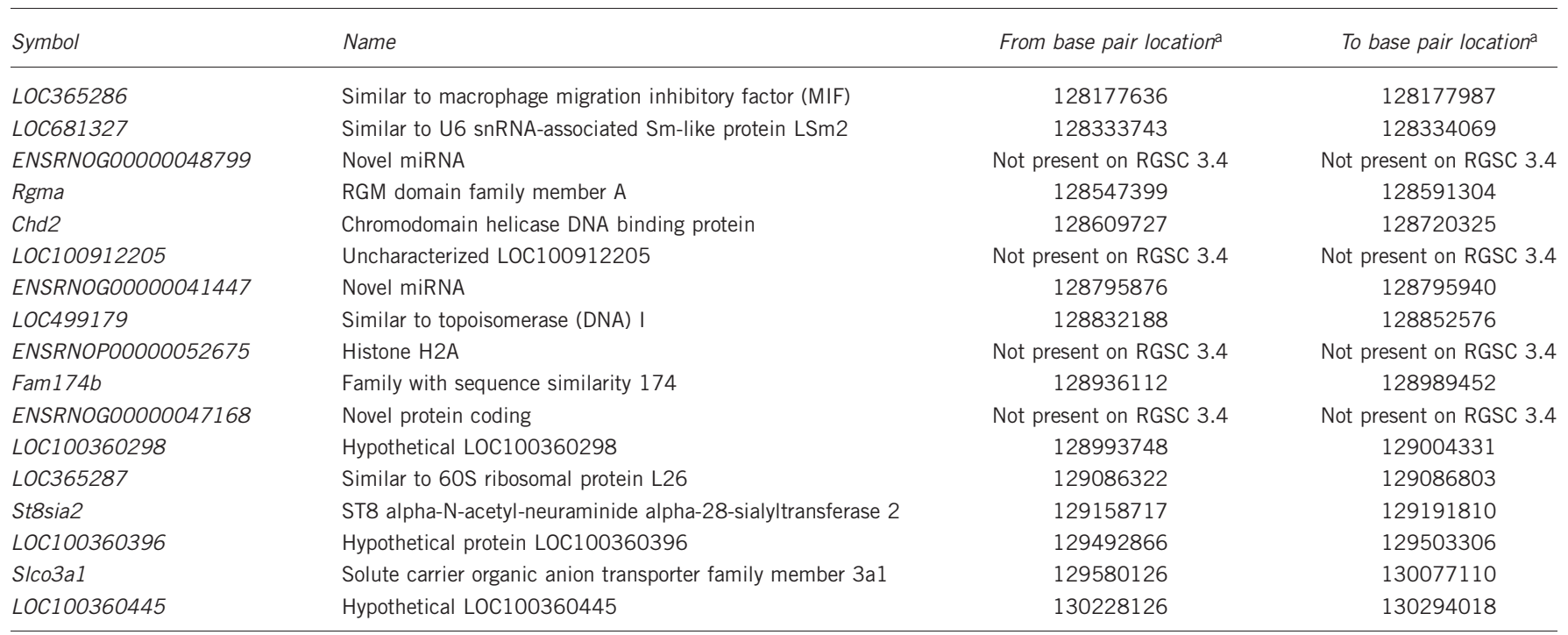

aBase pair locations on the rat genome were obtained from www.ensembl.org, RGSC 3.4.

Table 2b Annotations within QTL1b1a

\begin{tabular}{llr}
\hline Symbol & Name & From base pair location $^{\text {a }}$ \\
\hline Mctp2 & Multiple C2 domains transmembrane 2 & 127132844 \\
LOC365286 & Similar to macrophage migration inhibitory factor (MIF) & 127308205 \\
LOC681327 & Similar to U6 snRNA-associated Sm-like protein LSm2 & 128177636 \\
ENSRNOG00000048799 & Novel miRNA & 128333743 \\
Rgma & RGM domain family member A & Not present on RGSC 3.4 \\
\hline
\end{tabular}

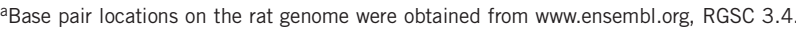

Table 3a Single-nucleotide polymorphisms between SS/Jr and LEW within the QTL regions

\begin{tabular}{lcccc}
\hline & Number of exonic & Number of & Number of & Number of 3' \\
Location & SNPs (protein-coding & intronic & intergenic & and 5' UTR \\
QTL1b1a & 4 (Mctp2) & SNPs & SNPs & SNPs \\
QTL1b1 & 0 & 42 & 1512 & 0 \\
\hline
\end{tabular}

Abbreviations: LEW, Lewis; QTL, quantitative trait loci; S, salt-sensitive; SNP, single-nucleotide polymorphism; UTR, untranslated region.

were fed a high-salt (2\%) diet were collected and analyzed by real-time PCR using a MyIQ ${ }^{\circledR}$ Real Time PCR Detection System (Bio-Rad, Hercules, CA, USA) as described previously. ${ }^{6}$ Gene-specific primers were designed using Primer3 (http://bioinfo.ut.ee/primer3/). Total RNA was reversed transcribed using an Oligo dT primer. cDNA was diluted and used as a template for quantitative PCR using SYBR Green. Using software provided by the MyIQ Real Time PCR Detection System, a first derivative melting curve analysis verified amplicon specificity. The quantification and normalization of relative gene expression was accomplished using the comparative threshold cycle $\left(\mathrm{C}_{\mathrm{T}}\right)$ method, or $\Delta \Delta \mathrm{C}_{\mathrm{T}} . \Delta \Delta \mathrm{C}_{\mathrm{T}}$ values were converted into ratios by $2^{-\Delta \Delta \mathrm{CT}}$ and averaged across biological replicates. The expression of the 'housekeeping' gene, ribosomal protein L36a (GenBank accession no. AA859783), was used for normalization, as this gene did not exhibit differential expression as previously shown. ${ }^{6}$
Table 3b Synonymous single-nucleotide variations between SS/Jr and LEW

\begin{tabular}{lccc}
\hline Location of SNP & $B N$ & LEW & S \\
\hline 127135212 & G & G & T \\
127267249 & C & C & T \\
127298426 & A & A & G \\
127300063 & G & G & A
\end{tabular}

Abbreviations: LEW, Lewis; S, salt-sensitive; SNP, single-nucleotide polymorphism.

Genomic sequencing and analysis

Genomic variant data between SS/Jr and LEW/NcrlBR were obtained from the Rat Genome Database (http://rgd.mcw.edu/).

\section{RESULTS}

The previously reported S.LEW(D1Mco36-D1Mco101) congenic substrain, which spanned the entire 7.39 Mb QTL1b1 region from 122.99 to $130.38 \mathrm{Mb}$, had a significant BP lowering effect of $-25 \mathrm{~mm} \mathrm{Hg}$ compared with the $\mathrm{S}$ rat. ${ }^{11}$ The homologous region to this rat genomic segment is shown in Figure 1 along with the BP QTL locations of the four linkage studies described in humans. To further fine-map QTL1b1, a panel of six congenic substrains was constructed and characterized (Figure 1). These congenic substrains were developed by introgressing LEW rat alleles into the genome of the 

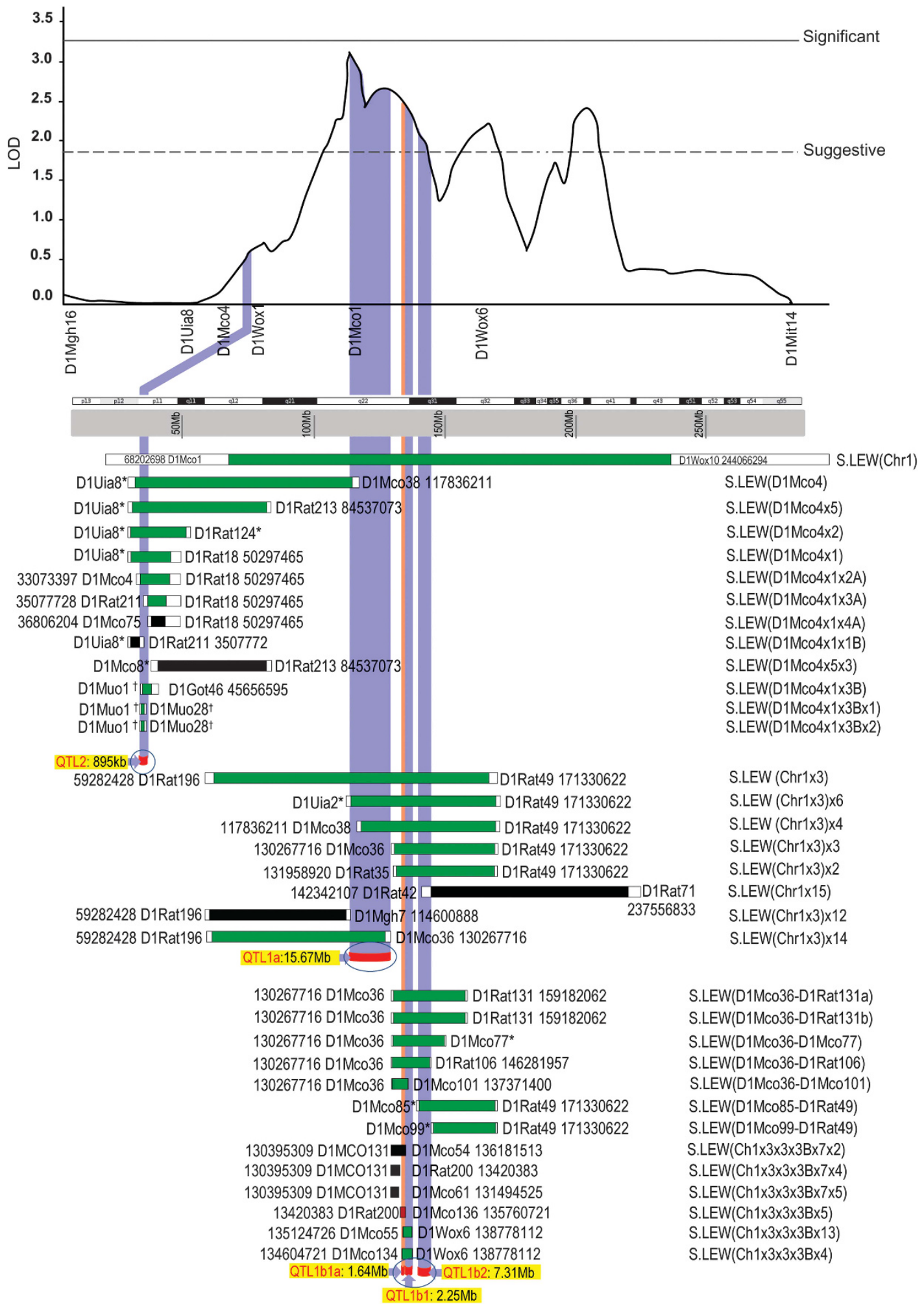

S.LEW(D1Mco36-D1Rat131a)

S.LEW(D1Mco36-D1Rat131b)

S.LEW(D1Mc036-D1Mc077)

S.LEW(D1Mco36-D1Rat106)

S.LEW(D1Mco36-D1Mc0101)

S.LEW(D1Mc085-D1Rat49)

S.LEW(D1Mc099-D1Rat49)

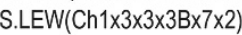

S.LEW $(\mathrm{Ch} 1 \times 3 \times 3 \times 3 \mathrm{~B} \times 7 \times 4)$

S.LEW $(\mathrm{Ch} 1 \times 3 \times 3 \times 3 \mathrm{~B} \times 7 \times 5)$

S.LEW(Ch1 $1 \times 3 \times 3 \times 3 \mathrm{~B} \times 5)$

S.LEW(Ch1 $13 \times 3 \times 3$ B $\times 13)$

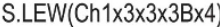

Figure 3 Comprehensive illustration of linkage and substitution mapping on RNO1 using S and LEW rats. The logarithm of odds plot for BP obtained by the study of an $\mathrm{F}_{2}(\mathrm{~S} \times \mathrm{LEW})$ population ${ }^{13}$ is shown at the top followed by the cytogenetic and physical maps of RNO1. End markers of congenic strains and their current physical locations (rat genome version 5.0) are flanking bars that represent congenic strains. The physical locations of markers were obtained from the rat genome database (http://www.rgd.mcw.edu). *Markers that were not mapped, location determined using nearby markers chosen using the limits of the congenic strains. 'Markers that were not located on RNO1, but are mapped to RNO1 based on our report. ${ }^{14}$ Green bars indicate that the congenic strain had a BP lowering effect, black bar indicates that the congenic strain had no BP effect and red bar indicates that the congenic strain had a BP increasing effect. All congenic strains except the ones currently reported are previously published.7,11-14 Vertical blue or orange lines ending in red arcs collectively depict all currently mapped QTL regions. The names of QTLs are highlighted in yellow along with their respective sizes. 
Dahl salt-sensitive (S) rat. Compared with the systolic BP of the $S$ rat, $198 \pm 8 \mathrm{mmHg}$, two of the new congenic substrains, S.LEW $(\mathrm{Ch} 1 \times 3 \times 3 \times 3 \mathrm{~B} \times 13)$ and S.LEW $(\mathrm{Ch} 1 \times 3 \times 3 \times 3 \mathrm{~B} \times 4)$, had significantly lower systolic BP of $157 \pm 6 \mathrm{mmHg} \quad(P<0.0001)$ and $155 \pm 16 \mathrm{mmHg}(P=0.003)$, respectively (Table 1$)$. These BP lowering effects observed by the tail-cuff method were corroborated using the telemetry method (Figure 2). The systolic, diastolic and mean BPs and pulse pressures of the congenic strains S.LEW $(\mathrm{Ch} 1 \times 3 \times 3 \times 3 \mathrm{~B} \times 13)$ and S.LEW $(\mathrm{Ch} 1 \times 3 \times 3 \times 3 \mathrm{~B} \times 4)$ were lower than that of the $S$ rat (Figure 2). The congenic strain S.LEW $(\mathrm{Ch} 1 \times 3 \times 3 \times 3 \mathrm{~B} \times 13)$ had a shorter LEW introgressed segment compared with S.LEW $(\mathrm{Ch} 1 \times 3 \times 3 \times 3 \mathrm{~B} \times 4)$ (Figure 1). Therefore, the location of BP QTL1b1 was interpreted to be within the congenic segment of S.LEW $($ Ch $1 \times 3 \times 3 \times 3 B \times 13)$ (Figure 1). Further, by comparing the lower ends of the congenic segments of the strains S.LEW(D1Mco36-D1Mco101) and S.LEW $($ Ch $1 \times 3 \times 3 \times 3 \mathrm{~B} \times 13)$, both of which demonstrated BP lowering effects, the genomic segment from D1Mco101 and D1Wox6 was eliminated. Therefore, the newly mapped location of QTL1b1 on RNO1 was within a 2.25-Mb region from 135.12 to $137.37 \mathrm{Mb}$. This represents a $70 \%$ improvement from the $7.39-\mathrm{Mb}$ region to which QTL1b1 was previously mapped. The newly mapped $2.25 \mathrm{Mb}$ QTL1b1 region contains eight genes and two predicted miRNA (Table 2a). There were no protein-coding variants within the exons of the eight genes or within the predicted miRNA.

Among the other newly developed congenic substrains shown in Figure 1 , the $\mathrm{BP}$ of three substrains, S.LEW $(\mathrm{Ch} 1 \times 3 \times 3 \times 3 \mathrm{~B} \times 7 \times 2)$, S.LEW $(\mathrm{Ch} 1 \times 3 \times 3 \times 3 \mathrm{~B} \times 7 \times 4)$ and S.LEW $(\mathrm{Ch} 1 \times 3 \times 3 \times 3 \mathrm{~B} \times 7 \times 5)$, was not significantly different from that of the $S$ rats. Note however that the systolic BP of the congenic substrain S.LEW $($ Ch $1 \times 3 \times 3 \times 3$ B $\times 5)$ $(198 \pm 6 \mathrm{~mm} \mathrm{Hg})$ was significantly higher by $20 \mathrm{~mm} \mathrm{Hg}$ compared with the systolic BP of the $S$ rat $(178 \pm 6 \mathrm{~mm} \mathrm{Hg}, P<0.0001)$. This result was consistent with BP measured by telemetry. Systolic, diastolic and mean BPs of S.LEW $(\mathrm{Ch} 1 \times 3 \times 3 \times 3 \mathrm{~B} \times 5)$ were higher than that of the $S$ rat (Figure 2). This newly identified locus, LEW alleles within which further increased systolic BP of the $S$ rat, was named QTL1b1a. QTL1b1a spans $1.64 \mathrm{Mb}$ and is located between 134.12 and $135.76 \mathrm{Mb}$. The QTL1bla region contains two protein-coding genes, Mctp2 and Rgma, and a novel predicted miRNA, ENSRNOG00000048799 (Table 2b). Genomic variants between SS/Jr and LEW/NcrlBR throughout the two QTL regions were obtained from the Rat Genome Database (http://rgd.mcw.edu/) and are shown in Supplementary Table S1. The majority of the variants in both QTL regions were located within either intergenic or intronic regions (Table 3a). However, within QTL1bla, four variants were confirmed in exonic regions of the protein-coding gene Mctp2. All were synonymous single-nucleotide polymorphisms. These four exonic SNPs were independently validated with genomic DNA of S, LEW and the congenic substrain S.LEW $(\mathrm{Ch} 1 \times 3 \times 3 \times 3 \mathrm{~B} \times 5)$ by capillary sequencing as described previously in the Materials and methods (Table 3b). Quantitative real-time PCR was performed on heart and kidney tissue to determine whether the expression of Mctp2 differed between the congenic substrain S.LEW $(\mathrm{Ch} 1 \times 3 \times 3 \times 3 \mathrm{~B} \times 5)$ and the $\mathrm{S}$ rat. The results indicated no significant difference in the expression of Mctp2 in either heart $(P=0.09)$ or kidney tissue $(P=0.70)$ (data not shown).

Figure 3 illustrates all iterations of substitution mapping performed on chromosome 1 by substituting LEW alleles into the $S$ rat genome. ${ }^{7,11-15}$ Including those identified by the current study, there are at least five independently functional BP QTLs within the originally detected logarithm of odds (LOD) plot. Of these, the underlying gene within QTL2 was previously validated using a genetically engineered mutant strain as a disintegrin-like metalloproteinase with thrombospondin motifs $16 .{ }^{14,15}$ QTL1a $^{12}$ could not be tracked further, as congenic strains with shorter segments did not demonstrate BP effects (unpublished data), and QTL1b is now resolved to three regions with candidate variants residing entirely outside the exonic regions of all three QTL regions.

\section{DISCUSSION}

Comparative mapping of human hypertension loci in rats provides an opportunity to discern loci that may participate in the regulation of $\mathrm{BP}$ in both species. ${ }^{16-18}$ While further genetic dissection is difficult in humans, substitution mapping in rats allows for further dissection of such loci. One such locus on human chromosome 15 and RNO1 is the subject of this study. This report is a new iteration of substitution mapping on RNO1, wherein a BP QTL region was previously located within a 7.39-Mb interval. Data collected from six new congenic substrains enabled further mapping of this $7.39 \mathrm{Mb}$ BP QTL to at least two shorter segments of 2.25 and $1.64 \mathrm{Mb}$. Further, it is interesting to note that although the overall effect of the LEW rat alleles underlying the QTL region was found to be protective based on linkage mapping results, there are LEW alleles within the QTL region that increase the $\mathrm{BP}$ of the S rat. The existence of such closely linked BP loci with opposing effects has been demonstrated in at least one other region of the rat genome, a region in which BP lowering effects were initially detected within a large QTL segment. ${ }^{10,19,20}$ The significance of such loci being closely linked within the genome is not clear but may suggest the complex nature of the genetic landscape of BP control in both rats and humans.

The current report represents a comprehensive mapping of the previously inferred $7.39 \mathrm{Mb}$ interval because the entire region was represented by the collective LEW allelic introgressed segments of the six new congenic strains. Genome sequencing of the QTL regions did not detect any non-synonymous exonic variants, but it did detect a large number of intronic and intergenic sequence variants. The mapping data and the sequencing data taken together suggest that the two newly mapped genomic intervals on chromosome 1 exert BP effects through variants that have functions beyond causing aminoacid substitutions within protein-coding genes. Detection of loci with evidence pointing to variants outside protein-coding genes as underlying candidates is not surprising because genome-wide association studies have similarly cataloged a large number of intergenic variants associated with several polygenic traits (http://www.genome.gov/ gwastudies/). Unlike variants within protein-coding genes or other non-coding genes, the lack of annotations encompassing these variants poses a significant problem for designing experiments to test the functional relevance of these variants to mechanisms underlying BP control. As several of the variants detected are within introns and intergenic regions, it is possible that these regions may interact with other chromosomal segments to varying degrees. Understanding the folding of the genome at this scale may be important in mammals where dispersed regulatory elements, such as enhancers and insulators, are involved in gene regulation. ${ }^{21}$ Therefore, beyond the next step of further mapping of the two QTLs, chromosomal conformation capture assays of such regions in question on other chromosomes could be contemplated. ${ }^{22,23}$

\section{ACKNOWLEDGEMENTS}

We would like to thank Prof John Paul Rapp for his review and constructive comments on the manuscript. This study was funded through research grants awarded to BJ by the National Heart Lung and Blood Institute of the National Institutes of Health (HL020176 and HL112641). 
1 Xu X, Yang J, Rogus J, Chen C, Schork N, Xu X. Mapping of a blood pressure quantitative trait locus to chromosome $15 q$ in a Chinese population. Hum Mol Genet 1999; 8: 2551-2555.

2 Xu X, Rogus JJ, Terwedow HA, Yang J, Wang Z, Chen C, Niu T, Wang B, Xu H, Weiss S, Schork NJ, Fang Z. An extreme-sib-pair genome scan for genes regulating blood pressure. Am J Hum Genet 1999; 64: 1694-1701.

3 Weder AB, Delgado MC, Zhu X, Gleiberman L, Kan D, Chakravarti A. Erythrocyte sodium-lithium countertransport and blood pressure: a genome-wide linkage study. Hypertension 2003; 41(3 Pt 2), 842-846.

4 Krushkal J, Ferrell R, Mockrin SC, Turner ST, Sing CF, Boerwinkle. E. Genome-wide linkage analyses of systolic blood pressure using highly discordant siblings. Circulation 1999; 99: 1407-1410.

5 Garrett MR, Meng H, Rapp JP, Joe B. Locating a blood pressure quantitative trait locus within $117 \mathrm{~kb}$ on the rat genome: substitution mapping and renal expression analysis. Hypertension 2005; 45: 451-459.

6 Joe B, Letwin NE, Garrett MR, Dhindaw S, Frank B, Sultana R, Verratti K, Rapp JP, Lee NH. Transcriptional profiling with a blood pressure QTL interval-specific oligonucleotide array. Physiol Genomics 2005; 23: 318-326.

7 Joe B, Garrett MR, Dene H, Rapp JP. Substitution mapping of a blood pressure quantitative trait locus to a $2.73 \mathrm{Mb}$ region on rat chromosome 1. J Hypertens 2003; 21: 2077-2084.

8 Lee SJ, Liu J, Westcott AM, Vieth JA, DeRaedt SJ, Yang S, Joe B, Cicila GT. Substitution mapping in Dahl rats identifies two distinct blood pressure quantitative trait loci within 1.12- and 1.25-mb intervals on chromosome 3. Genetics 2006; 174: 2203-2213.

9 Saad Y, Garrett MR, Manickavasagam E, Yerga-Woolwine S, Farms P, Radecki T, Joe B. Fine-mapping and comprehensive transcript analysis reveals nonsynonymous variants within a novel $1.17 \mathrm{Mb}$ blood pressure QTL region on rat chromosome 10. Genomics 2007; 89: 343-353.

10 Saad Y, Yerga-Woolwine S, Saikumar J, Farms P, Manickavasagam E, Toland EJ, Joe B. Congenic interval mapping of RNO10 reveals a complex cluster of closely-linked genetic determinants of blood pressure. Hypertension 2007; 50: 891-898.

11 Toland EJ, Saad Y, Yerga-Woolwine S, Ummel S, Farms P, Ramdath R, Frank BC, Lee $\mathrm{NH}$, Joe $\mathrm{B}$. Closely linked non-additive blood pressure quantitative trait loci. Mamm Genome 2008; 19: 209-218.

12 Saad Y, Garrett MR, Rapp JP. Multiple blood pressure QTL on rat chromosome 1 defined by Dahl rat congenic strains. Physiol Genomics 2001; 4: 201-214.
13 Garrett MR, Dene H, Walder R, Zhang QY, Cicila GT, Assadnia S, Deng AY, Rapp JP. Genome scan and congenic strains for blood pressure QTL using Dahl salt-sensitive rats. Genome Res 1998; 8: 711-723.

14 Joe B, Saad Y, Dhindaw S, Lee NH, Frank BC, Achinike OH, Luu TV, Gopalakrishnan K, Toland EJ, Farms P, Yerga-Woolwine S, Manickavasagam E, Rapp JP, Garrett MR, Coe D, Apte SS, Rankinen T, Pérusse L, Ehret GB, Ganesh SK, Cooper RS, O'Connor A, Rice T, Weder AB, Chakravarti A, Rao DC, Bouchard C. Positional identification of variants of Adamts16 linked to inherited hypertension. Hum Mol Genet 2009; 18 : 2825-2838.

15 Gopalakrishnan K, Kumarasamy S, Abdul-Majeed S, Kalinoski AL, Morgan EE, Gohara AF, Nauli SM, Filipiak WE, Saunders TL, Joe B. Targeted disruption of Adamts 16 gene in a rat genetic model of hypertension. Proc Natl Acad Sci USA 2012; 109: 20555-20559.

16 Stoll M, Jacob HJ. Genetic rat models of hypertension: relationship to human hypertension. Curr Hypertens Rep 2001; 3: 157-164.

17 Stoll M, Kwitek-Black AE, Cowley AW Jr, Harris EL, Harrap SB, Krieger JE, Printz MP, Provoost AP, Sassard J, Jacob HJ. New target regions for human hypertension via comparative genomics. Genome Res 2000; 10: 473-482.

18 Shimoyama M, Smith JR, Hayman T, Laulederkind S, Lowry T, Nigam R, Petri V, Wang SJ, Dwinell M, Jacob HRGD Team. RGD: a comparative genomics platform. Hum Genomics 2011; 5: 124-129.

19 Gopalakrishnan K, Kumarasamy S, Yan Y, Liu J, Kalinoski A, Kothandapani A, Farms P, Joe $B$. Increased expression of Rififylin in $A<330 \mathrm{~Kb}$ congenic strain is linked to impaired endosomal recycling in proximal tubules. Front Genet 2012; 3: 138.

20 Gopalakrishnan K, Morgan EE, Yerga-Woolwine S, Farms P, Kumarasamy S, Kalinoski A, Liu X, Wu J, Liu L, Joe B. Augmented Rififylin is a risk factor linked to aberrant cardiomyocyte function, short-QT interval and hypertension. Hypertension 2011; 57: 764-771.

21 Hagege H, Klous P, Braem C, Splinter E, Dekker J, Cathala G, de Laat W, Forné T. Quantitative analysis of chromosome conformation capture assays (3C-qPCR). Nat Protoc 2007; 2: 1722-1733.

22 Smits BM, Haag JD, Rissman AI, Sharma D, Tran A, Schoenborn AA, Baird RC, Peiffer DS, Leinweber DQ, Muelbl MJ, Meilahn AL, Eichelberg MR, Leng N, Kendziorski C, John MC, Powers PA, Alexander CM, Gould MN. The gene desert mammary carcinoma susceptibility locus Mcsla regulates Nr2f1 modifying mammary epithelial cell differentiation and proliferation. PLOS Genet 2013; 9: e1003549.

23 Schierding W, Cutfield WS, O'Sullivan. JM. The missing story behind Genome Wide Association Studies: single nucleotide polymorphisms in gene deserts have a story to tell. Front Genet 2014; 5: 39.

Supplementary Information accompanies the paper on Hypertension Research website (http://www.nature.com/hr) 